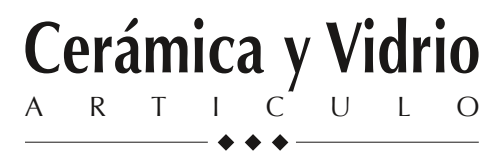

\title{
Caracterización química y mineralógica de refractarios de $\mathrm{Al}_{2} \mathrm{O}_{3}-\mathrm{MgO}-\mathrm{C}$
}

\author{
P. ORTEGA ${ }^{1}$, M. J. VELASCO ${ }^{1}$, V. MUÑOZ ${ }^{2}$, A. G. TOMBA MARTíNEZ ${ }^{2}$, P. PENA ${ }^{1}$ \\ (1) Instituto de Cerámica y Vidrio ICV-CSIC, Kelsen 5, 28049 - Madrid, España \\ (2) Instituto de Investigaciones en Ciencia y Tecnología de Materiales (INTEMA) \\ CONICET-Facultad de Ingeniería/Universidad Nacional de Mar del Plata \\ Av. J.B. Justo 4302 (7600) Mar del Plata, Argentina
}

\begin{abstract}
Los refractarios de $\mathrm{Al}_{2} \mathrm{O}_{3}-\mathrm{MgO}-\mathrm{C}$, de gran interés tecnológico por sus excelentes propiedades, se utilizan mayoritariamente en las industrias del hierro y el acero. Estos materiales están constituidos por distintas calidades de corindón, periclasa y grafito a los que se añaden pequeñas cantidades de metales y un polímero que actúa como ligante.

En este trabajo establece una metodología para la caracterización química y mineralógica de refractarios de $\mathrm{Al}_{2} \mathrm{O}_{3}-\mathrm{MgO}_{\mathrm{O}} \mathrm{C}$. $\mathrm{Los}$ resultados del análisis químico realizado por fluorescencia de rayos $\mathrm{X}$, espectroscopia de emisión de plasma y gravimetría, junto con la información cualitativa acerca de las fases cristalinas (obtenida por difracción de rayos X), y el conocimiento de las materias primas con las que están formulados (obtenido por análisis térmico diferencial y termogravimétrico y microscopia óptica de luz reflejada), se han utilizado para conocer la composición de los refractarios. Los datos obtenidos por las diferentes técnicas dan validez a la metodología desarrollada.
\end{abstract}

Palabras clave: refractarios $\mathrm{Al}_{2} \mathrm{O}_{3}-\mathrm{MgO}-\mathrm{C}$, microscopia óptica, difracción de rayos $\mathrm{X}$, fluorescencia de Rayos X, espectroscopia de emisión de plasma.

\section{Chemical analysis of $\mathrm{Al}_{2} \mathrm{O}_{3}-\mathrm{MgO}-\mathrm{C}$ refractories}

The $\mathrm{Al}_{2} \mathrm{O}_{3}-\mathrm{MgO}-\mathrm{C}$ refractories, of great technological interest for its excellent properties, are used in a wide range of furnacelining applications such as iron and steel. These materials are composed of various grades of alumina, magnesia, graphite and metallic additives, which are added to a resin that acts as binder. The variety of components oxides, metals and polymers makes the study of these refractories a complex task. Considering this diversity to characterize these materials has been used several techniques: X-ray fluorescence, plasma emission spectroscopy and gravimetry, complemented by X-ray diffraction, differential thermal analysis and thermogravimetry and reflected light optical microscopy.

This paper provides a methodology for chemical and mineralogical characterization of these refractory materials. The results of chemical analysis together with the qualitative information on the crystalline phases and the raw materials with which they are formulated have been used to quantify the composition of the refractories using rational analysis. The data obtained by the different techniques validate the methodology developed.

Keywords: $\mathrm{Al}_{2} \mathrm{O}_{3}-\mathrm{MgO}-\mathrm{C}$ refractories, optical microscopy, X-ray diffraction, X-ray fluorescence, plasma emission spectroscopy.

\section{INTRODUCCIÓN}

Desde los años 80, los refractarios de $\mathrm{MgO}-\mathrm{C}$ y de $\mathrm{Al}_{2} \mathrm{O}_{3}$ $\mathrm{MgO}-\mathrm{C}$ (AMC) (1-7) se utilizan cada vez más frecuentemente en las industrias del hierro y el acero, lo que ha dado lugar a una significativa mejora en los procesos metalúrgicos. Más específicamente, los ladrillos de $\mathrm{MgO}-\mathrm{C}$ se usan como revestimiento en los hornos eléctricos de arco y en línea de escoria de las cucharas de colada, mientras que los de $\mathrm{Al}_{2} \mathrm{O}_{3}$ $\mathrm{MgO}-\mathrm{C}$ se emplean en paredes y fondos de cuchara. En las últimas décadas, la situación cada vez más competitiva, con una fuerte tendencia a mejorar la calidad del acero, ha dado lugar a cambios significativos en los procesos metalúrgicos y en las condiciones de trabajo de las cucharas. Esto ha dado lugar a mejoras en sus características y propiedades para optimizar su comportamiento en servicio.
La adición de grafito a los refractarios de corindón $\left(\alpha-\mathrm{Al}_{2} \mathrm{O}_{3}\right)$, periclasa $(\mathrm{MgO})$, o a mezclas de los mismos mejora su comportamiento en uso, esto se debe a que los refractarios con grafito (C) no son mojados por metales ni escorias, lo que dificulta la penetración de estos fundidos a través de los poros abiertos de estos materiales (8). Así mismo, estos refractarios presentan una resistencia al choque térmico óptima consecuencia de la baja expansión térmica y elevada conductividad térmica (9) que les aporta el grafito. Por otro lado, el grafito puede absorber esfuerzos mecánicos y/o térmicos debido a la flexibilidad de su estructura (10).

Así mismo, hay que considerar que el grafito se oxida fácilmente a alta temperatura, por lo que la resistencia a la oxidación de estos materiales es un factor limitante de su 
comportamiento en servicio. Para aumentar la resistencia a la oxidación del grafito de estos materiales, se añaden cantidades controladas (0-5\% en peso) de "antioxidantes": comúnmente metales $(\mathrm{Al}, \mathrm{Si}, \mathrm{Mg})$ y sus aleaciones (Al-Mg) y con menos frecuencia, carburos $\left(\mathrm{SiC}, \mathrm{B}_{4} \mathrm{C}\right)$ y boruros $\left(\mathrm{CaB}_{6}\right.$, $\mathrm{ZrB}_{2}$ ). En servicio, estos aditivos reaccionan con el $\mathrm{C}$ y con los gases presentes en los poros del material $\left(\mathrm{O}_{2^{\prime}} \mathrm{CO} \mathrm{y} / \mathrm{o}\right.$ $\mathrm{N}_{2}$ ) formando compuestos (carburos, óxidos y nitruros); estas reacciones contribuyen a evitar la pérdida del grafito por oxidación. Los nuevos compuestos se depositan como plaquetas y/o 'whiskers' que rellenan los poros y / o reaccionan con las partículas cerámicas (alúmina y magnesia) formando otras fases sólidas; ambos procesos, además, mejoran las propiedades mecánicas del material $(11,12)$.

Los refractarios de $\mathrm{Al}_{2} \mathrm{O}_{3}-\mathrm{MgO}-\mathrm{C}$ (AMC) están comúnmente constituidos por partículas con una granulometría formada por una fracción gruesa (6 a $1 \mathrm{~mm})$, una media (1 mm $-120 \mu \mathrm{m})$ y una fina $(<120 \mu \mathrm{m})$. Las fracciones media y gruesa están constituidas por agregados de alúmina (alúmina tabular, alúmina electrofundida y / o bauxitas) y magnesia (calcinada a muerte 'sinter' y/o electrofundida). La fracción fina está formada por partículas de corindón, periclasa (preferentemente electrofundida), escamas de grafito y aluminio. Este tipo de refractarios no se sinterizan durante su fabricación, sino que la unión entre las partículas se realiza con una fase polimérica (13), brea o resina, "templada" a $100-300^{\circ} \mathrm{C}$ o sin "templar".

Durante la utilización de estos materiales, tienen lugar reacciones que involucran al corindón, la periclasa y el aluminio, fundamentalmente en la fracción fina, que dan lugar a la formación de espinela $\left(\mathrm{MgAl}_{2} \mathrm{O}_{4}\right)$ por diversos mecanismos (14-17). Esta reacción está acompañada de procesos de expansión que pueden contrarrestar el desgaste en las juntas entre los ladrillos refractarios. Además, esta expansión también produce microfisuración debido a la diferencia entre los coeficientes de expansión térmica del producto y los reactivos, la cual podría dar lugar a mecanismos de reforzamiento; sin embargo, estas microfisuras también pueden convertirse en puntos de ingreso de agentes químicamente agresivos. Por estos motivos, la reacción de formación de espinela tiene que ser controlada y en consecuencia, el contenido de $\mathrm{MgO}$ debe ser optimizado (3).

Los trabajos de investigación utilizando refractarios comerciales son poco habituales porque, en general, no es posible diseñar ni controlar la composición química y/o microestructural de los materiales en estudio. Pero si se dispone de series de refractarios comerciales y de una buena caracterización de éstos, se podrán generar conocimientos básicos sobre las relaciones composición-microestructurapropiedades, con los cualesse podrá explicar el comportamiento en servicio de un material específico y obtener relaciones para mejorar el diseño de los materiales.

La caracterización de los refractarios de alúmina-magnesiagrafito, que contienen fases elementales ( $\mathrm{C} \mathrm{y} \mathrm{Al}$ ), óxidos inorgánicos $\left(\mathrm{MgO}, \mathrm{Al}_{2} \mathrm{O}_{3}\right)$, una fase orgánica (aglutinante), diversos aditivos y las impurezas de todos ellos, es compleja y requiere una planificación cuidadosa de los experimentos.

La metodología para el análisis químico de cada uno de los componentes de estos materiales ha sido establecida en un gran número de estudios [18], pero sólo desde un conocimiento profundo del comportamiento térmico de estos materiales multicomponentes (datos de análisis térmico diferencial y termogravimétrico), de su composición mineralógica (información obtenida por difracción de rayos X) y del tipo y composición de la fase orgánica (espectroscopias IR y Raman) entre otros se puede establecer su composición exacta. De Aza y col [19], utilizando esta estrategia, abordaron el análisis químico completo de un material de magnesiagrafito y para ello realizaron los análisis químicos por ICPOES. En un artículo posterior abordaron el análisis global del material utilizando conjuntamente los resultados del análisis químico y los obtenidos mediante difracción de rayos $\mathrm{X}$ utilizando la técnica de Rietveld [20].

C. Baudin y col. [11,12] realizaron los análisis de doce tipos de ladrillos comerciales de magnesia-grafito-aluminio metálico por Espectroscopia de Emisión por Plasma (ICPOES), análisis térmico diferencial (ATD) y difracción de rayos $X(D R X)$. En estas investigaciones, la muestra a analizar una vez calcinada para eliminar el material orgánico y quemar el grafito se funde con carbonato alcalino y tetraborato sódico dentro de un crisol de platino y la masa fundida se disuelve en ácido clorhídrico. Para determinar la cantidad de carbono realizaron análisis termogravimétrico (TG).

Jecko y Ravaine [21] realizaron análisis de refractarios de magnesia-carbono, alúmina-carburo de silicio-carbono, carburo de silicio, nitruro de silicio, nitruro de boro, y sialones utilizando los métodos convencionales usados en la caracterización de refractarios tradicionales como fluorescencia de rayos X (FRX), AAS y análisis elemental. Sin embargo, este autor hizo una primera aproximación al análisis de los elementos metálicos ( $\mathrm{Si}, \mathrm{Al})$ aunque sin aportar datos cuantitativos.

El objetivo del presente artículo es establecer una metodología experimental para la caracterización química y mineralógica de refractarios comerciales de $\mathrm{Al}_{2} \mathrm{O}_{3}-\mathrm{MgO}-\mathrm{C}$ utilizados como revestimiento en paredes y fondo de cucharas de acería, en contacto con el metal fundido. La metodología implementada ha requerido el uso conjunto de diferentes técnicas: a) análisis químico (por FRX e ICP-OES), b) análisis mineralógico (por DRX), c) análisis térmico (por ATD-TG) y d) análisis microestructural (por MOLR). Finalmente, utilizando el análisis racional para interpretar los resultados del análisis químico y la información obtenida por ATD-TG, DRX y MOLR, se ha estimado el contenido de las fases mineralógicas mayoritarias.

\section{PROCEDIMIENTO EXPERIMENTAL}

\subsection{Materiales}

Se han analizado tres refractarios comerciales de $\mathrm{Al}_{2} \mathrm{O}_{3}$ $\mathrm{MgO}-\mathrm{C}$ del mismo proveedor, que se han denominado $\mathrm{AMC1}$, AMC2 y AMC3. Según su hoja técnica, estos refractarios se han formulado con distintas proporciones de agregados de alúmina tabular y/o bauxita, magnesia sinterizada y grafito y se han aglomerado con resina.

\subsection{Técnicas utilizadas}

\subsubsection{DIFRACCIÓN DE RAYOS X}

El análisis mineralógico se ha realizado por difracción de rayos $X$ sobre muestras en polvo $(<75 \mu \mathrm{m})$ en un difractómetro Brucker Advanced DV8, usando radiación $\mathrm{K} \alpha$ de $\mathrm{Cu}(1,5418 \AA ̊ \AA)$, monocromador primario $\mathrm{K}_{\alpha 1}$, a un voltaje 
de $40 \mathrm{kV}$ y una corriente de $40 \mathrm{~mA}$ y detector multicanal ("ojo de lince"). El registro de los difractogramas se ha realizado en el intervalo angular $10-70{ }^{\circ} 2 \theta$, con un tamaño de paso de $0,009^{\circ}$ y un tiempo de adquisición por paso de $23 \mathrm{~s}$ para los materiales originales y de $0,05^{\circ}$ y $20 \mathrm{~s}$ para los materiales tratados térmicamente.

\subsubsection{ANÁLISIS TÉRMICO}

Los análisis térmico diferencial (ATD) y termogravimétrico de los materiales se han realizado sobre muestras en polvo (< $75 \mu \mathrm{m})$ en un equipo Shimadzu DTA-50 y TGA-50, a una velocidad de calentamiento de $10{ }^{\circ} \mathrm{C} / \mathrm{min}$, hasta $1200{ }^{\circ} \mathrm{C}$, en flujo de aire seco. Se ha utilizado $\alpha$-alúmina como material de referencia y un crisol de Pt.

\subsubsection{MICROSCOPIA ÓPTICA}

El estudio microestructural de los refractarios se ha realizado sobre secciones pulidas (hasta una rugosidad $<1$ $\mu \mathrm{m})$ de muestras extraídas de cada ladrillo y con los poros abiertos rellenos de una resina epoxi. La identificación de las fases se ha efectuado por microscopia óptica de luz reflejada (MOLR), empleado un estereomicroscopio Carl Zeiss Axiophot.

\subsubsection{FLUORESCENCIA DE RAYOS X}

El análisis químico de los materiales se ha realizado mediante la técnica de Fluorescencia de Rayos $X$ por dispersión de longitudes de onda, utilizando un equipo PANalytical,MagicX (PW-2424), con un tubo de RX de ánodo de Rh (SUPER SHARP) y generador de 2,4 KW.

\subsubsection{ESPECTROSCOPIA DE EMISIÓN POR PLASMA}

Para la cuantificación del aluminio metálico se ha utilizado la técnica de Espectroscopia de Emisión por Plasma en un equipo Thermo Jarrell Ash IRIS Advantage Axial Plasma, con un generador de radiofrecuencia de $40,68 \mathrm{MHz}$ y un detector de estado sólido (CID, Charge Injection Device). El aluminio se midió en la longitud de onda $\lambda$ : 396.153nm

\subsection{Preparación de muestras}

El análisis mineralógico, térmico y químico de los materiales estudiados se ha realizado en muestras representativas de cada refractario, extraídas de piezas sin utilizar. Para la preparación de las muestras se han triturado aproximadamente $200 \mathrm{~g}$ de ladrillo que se han cuarteado hasta obtener 20-25 g de polvo grueso. Posteriormente, se ha reducido el tamaño de partícula a $<75 \mu \mathrm{m}$ (200 mallas) en un molino planetario Planetary- Micro Mill "Pulverisette 7" Fritsch, a una velocidad de $595 \mathrm{rpm}$.

El análisis por FRX se ha realizado en muestras preparadas en forma de perla, empleando una perladora Perl'X3 de Philips y un crisol de Pt-Au. Las perlas se han obtenido por fusión a $1050{ }^{\circ} \mathrm{C}$ de una mezcla homogénea de 0,3000 g de la muestra problema en polvo $(<75 \mu \mathrm{m})$ con $5,5000 \mathrm{~g}$ de $\mathrm{Li}_{2} \mathrm{~B}_{4} \mathrm{O}_{7}$ anhidro y $0,06 \mathrm{~g}$ de $\mathrm{LiBr}$ (despegante). El fundido se ha colado en un molde de la misma composición que el crisol, resultando una perla homogénea y transparente.
En el caso de muestras que contienen Al en forma metálica, el crisol de Pt-Au puede dañarse durante la preparación de la perla por aleación con este metal. Por otra parte, a la temperatura de fusión de la perla $\left(1050{ }^{\circ} \mathrm{C}\right)$, tanto la resina como el grafito se oxidan $\left(\mathrm{C}(\mathrm{s})+3 / 2 \mathrm{O}_{2}(\mathrm{~g}) \rightarrow \mathrm{CO}_{2}(\mathrm{~g})\right)$ por lo que formarían burbujas de $\mathrm{CO}_{2}$ que dificultarían el posterior análisis. Por este motivo, es necesario oxidar el $\mathrm{Al}$, el grafito y el polimero presente en las muestras a $1100{ }^{\circ} \mathrm{C}$.

Por otra parte, para la cuantificación del Al metálico, se ha separado esta especie del resto de los componentes. Sobre $0,200 \mathrm{~g}$ de muestra en polvo $(<75 \mu \mathrm{m})$ sometida a tratamiento térmico a $600{ }^{\circ} \mathrm{C}$ se ha realizado un ataque ácido a ebullición con $\mathrm{HCl}$ (1:1), durante 1 hora.

Durante este proceso, sólo el aluminio metálico reacciona con el ácido según: $2 \mathrm{Al}^{0}(\mathrm{~s})+6 \mathrm{H}^{+} \rightarrow 2 \mathrm{Al}^{3+}+3 \mathrm{H}_{2}(\mathrm{~g})$. El aluminio se ha determinado a partir de la disolución resultante por ICPOES.

La cantidad de grafito y resina presentes en los refractarios no puede determinarse por FRX ni por ICP-OES. Los contenidos de estos compuestos se han cuantificado mediante la determinación de la pérdida de peso (gravimetría) de muestras en polvo tratadas térmicamente con el objetivo de eliminar selectivamente cada uno ellos.

\subsubsection{TRATAMIENTOS TÉRMICOS}

Los tratamientos térmicos se han realizado en aire, empleando un horno eléctrico de cámara con elementos calefactores de $\mathrm{SiC}$ sobre $10 \mathrm{~g}$ de polvo $(<75 \mu \mathrm{m})$. Las temperaturas de los tratamientos se han establecido teniendo en cuenta los resultados del ATD-TG (apartado 3.3).

Para la preparación de las muestras para el análisis por ICP-OES y FRX, muestras en polvo de cada composición se trataron a $600{ }^{\circ} \mathrm{C}$ y $1100{ }^{\circ} \mathrm{C}$ respectivamente. Para eliminar la resina y el grafito fueron necesarias un total de $34 \mathrm{~h}$ a 600 ${ }^{\circ} \mathrm{C}$. Para oxidar el aluminio metálico a alúmina se necesitaron $22 \mathrm{~h}$ a $1100{ }^{\circ} \mathrm{C}$, lo que se ha constatado mediante el análisis mineralógico comparativo por DRX de los polvos originales y de los tratados térmicamente.

Para la cuantificación de la resina y el grafito por gravimetría, se han realizado tratamientos térmicos de $4 \mathrm{~h}$ a 500 y $800^{\circ} \mathrm{C}$, respectivamente, sobre polvo con un tamaño de grano $<75 \mu \mathrm{m}$. La efectividad de la eliminación selectiva de los componentes se ha confirmado por comparación de los análisis termogravimétricos del polvo original y del tratado térmicamente.

\subsubsection{REACTIVOS DE ANÁLISIS}

Para el análisis químico de los refractarios se han empleado los siguientes reactivos: Tetraborato de Litio $\left(\mathrm{Li}_{2} \mathrm{~B}_{4} \mathrm{O}_{7}\right.$, Merck para análisis), bromuro de litio (BrLi, Aldrich, pureza > 99.995\% acido clorhídrico $\left(\mathrm{HCl}, \mathrm{d}=1.19\right.$ g.ml ${ }^{-1}$, Merck grado RA), una solución patrón de aluminio de $1 \mathrm{~g} \cdot \mathrm{l}^{-1} \mathrm{y}$ agua desionizada con una resistividad menor que $18 \mathrm{M} \Omega$. 


\section{RESULTADOS Y DISCUSION}

\subsection{Composición mineralógica}

En la Figura 1 se muestran los difractogramas de los tres materiales analizados y en la Tabla I se indica la composición mineralógica resultante. Las fases mayoritarias en los tres refractarios son: corindón $\left(\alpha-\mathrm{Al}_{2} \mathrm{O}_{3}\right.$; Ficha 43-1484), periclasa (MgO; Ficha 43-1022) y grafito (C; Ficha 41-1487). En los tres materiales se han detectado pequeñas cantidades de aluminio metálico picos a $2 \Theta=38,5$ y a 44,7 ( $\mathrm{Al}$; Ficha 04-0787) y trazas de hematita picos a $2 \Theta=33,2$ y a 35,6 $\left(\mathrm{Fe}_{2} \mathrm{O}_{3}\right.$; Ficha 33-0664). En AMC3 también se han identificado: mullita $\left(3 \mathrm{Al}_{2} \mathrm{O}_{3} \cdot 2 \mathrm{SiO}_{2}=\mathrm{Al}_{6} \mathrm{Si}_{2} \mathrm{O}_{13}\right.$; Ficha 84-1205), titanato de aluminio $\left(\mathrm{Al}_{2} \mathrm{O}_{3} \cdot \mathrm{TiO}_{2}=\mathrm{Al}_{2} \mathrm{TiO}_{5} ;\right.$ Ficha $\left.41-0258\right)$, rutilo $\left(\mathrm{TiO}_{2}\right.$; Ficha 21-1276) y pseudobroquita $\left(\mathrm{Fe}_{2} \mathrm{O}_{3} \cdot \mathrm{TiO}_{2}=\mathrm{Fe}_{2} \mathrm{TiO}_{5}\right.$; Ficha 41-1432); la presencia de estas fases indica que este refractario ha sido formulado con bauxita.

TABLA I: ANÁLISIS MINERALÓGICO POR DRX.

\begin{tabular}{|c|c|c|c|}
\hline Fases & AMC1 & AMC2 & AMC3 \\
\hline Corindón $\left(\mathrm{a}-\mathrm{Al}_{2} \mathrm{O}_{3}\right)$ & mayoritario & mayoritario & mayoritario \\
\hline Periclasa $(\mathrm{MgO})$ & intermedia & intermedia & intermedia \\
\hline Grafito $(\mathrm{C})$ & intermedia & intermedia & intermedia \\
\hline Aluminio $(\mathrm{Al})$ & minoritario & minoritario & minoritario \\
\hline Hematita $\left(\mathrm{Fe}_{2} \mathrm{O}_{3}\right)$ & trazas & trazas & trazas \\
\hline Mullita $\left(3 \mathrm{Al}_{2} \mathrm{O}_{3} \cdot 2 \mathrm{SiO}_{2}\right)$ & - & - & trazas \\
\hline Titanato de aluminio $\left(\mathrm{Al}_{2} \mathrm{TiO}_{5}\right)$ & - & - & trazas \\
\hline Rutilo $\left(\mathrm{TiO}_{2}\right)$ & - & - & trazas \\
\hline Pseudobrooquita $\left(\mathrm{Fe}_{2} \mathrm{TiO}_{5}\right)$ & - & - & trazas \\
\hline
\end{tabular}

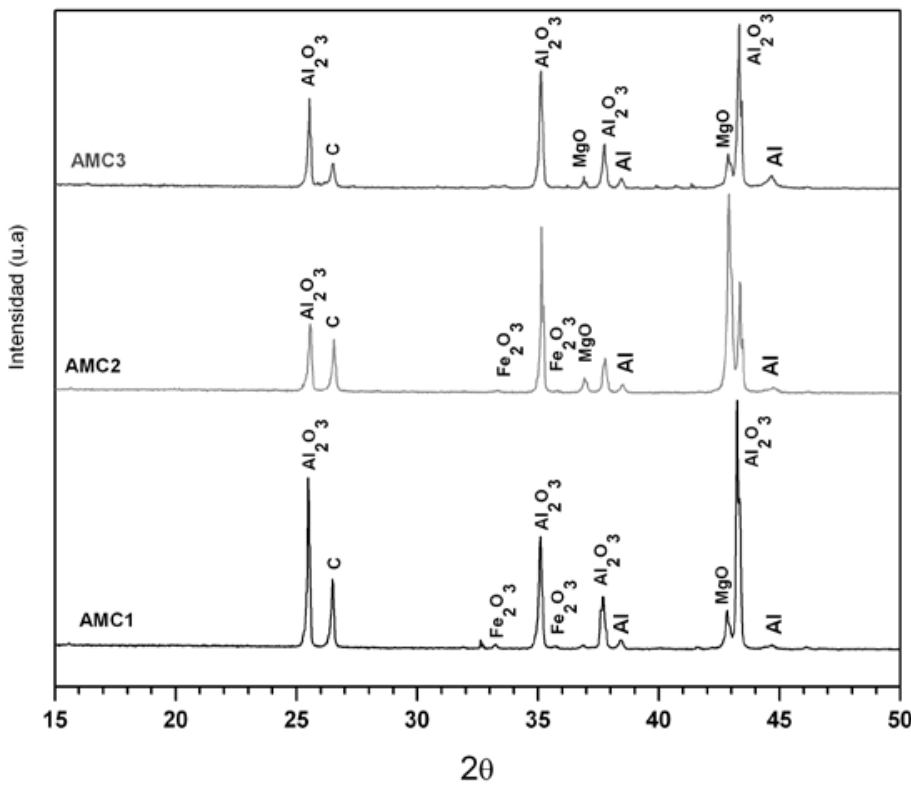

Figura 1. Difractogramas de polvo típicos de los refractarios estudiados.
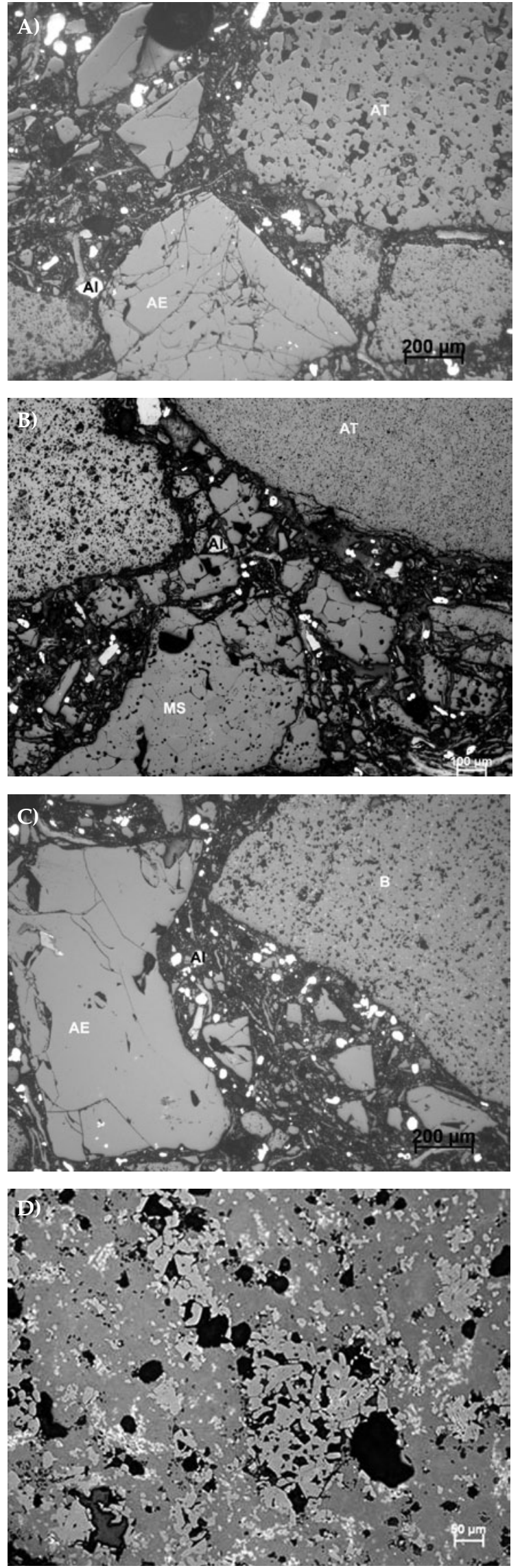

Figura 2. Imágenes de microscopía óptica mostrando las microestructuras típicas de los materiales refractarios: a) AMC1; b) AMC2; c) AMC3 y d) detalle de un agregado de bauxita de la muestra AMC3. Alúmina tabular $=\mathrm{AT}$, alúmina electrofundida $=\mathrm{AE}$, bauxita $=\mathrm{B}$. 


\subsection{Microestructura}

En la Figura 2 se muestran micrografías obtenidas por microscopía óptica de las microestructuras típicas de los tres refractarios. El análisis microscópico ha permitido identificar la presencia de:

- Agregados de 6-1 mm de alúmina de diferentes tipos: a) alúmina tabular (AT) de elevada pureza, b) alúmina electrofundida (AE) marrón con impurezas de $\mathrm{Fe}$ y $\mathrm{Ti}$ (hematita; titanatos de $\mathrm{Fe} / \mathrm{Al}$, corindón), y fase vítrea; c) de bauxita (B) sólo en el refractario AMC3; en éste último caso se identificaron agregados formados por cristales hexagonales característicos de corindón, de color gris claro y con un fuerte relieve, agujas de color gris típicas de mullita y/o cristales de color blanco de característicos de titanatos de Fe/ $\mathrm{Al}$;

- Agregados con una granulometría correspondiente a la fracción media - fina de magnesia sinterizada (MS).

- Grafito en escamas (G) (con un tamaño de 100 -500 $\mu \mathrm{m}$ de longitud y $10 \mu \mathrm{m}$ de anchura).

- Granos redondos con brillo metálico y un tamaño de 10-200 micras de aluminio metálico (Al).

Las distintas fases minoritarias identificadas por DRX son consistentes con las impurezas que suelen acompañar a los principales componentes de los refractarios (bauxita, periclasa y alúmina electrofundida) identificados en el análisis microscópico. A partir de los resultados del análisis por DRX y microscópico se estimó la formulación de cada refractario, indicada en la Tabla II.

\subsection{Comportamiento térmico}

La presencia simultánea de resina, grafito (C), oxidos $\left(\mathrm{Al}_{2} \mathrm{O}_{3}\right.$ y $\left.\mathrm{MgO}\right)$ y una fase metálica $\left(\mathrm{Al}^{0}\right)$, que sufren transformaciones de diferente índole a elevada temperatura, permite caracterizar los refractarios estudiados por ATD-TG, a través de la identificación de las reacciones y pérdidas de peso que tienen lugar durante el calentamiento. En este estudio en particular, el uso de estas técnicas permite, además, establecer las temperaturas de los tratamientos térmicos adecuadas para la oxidación selectiva de ciertos componentes (resina, grafito y aluminio metálico).

Los termogramas de los tres refractarios en aire, que se muestran en la Figura 3, confirman la presencia de una fase orgánica tipo resina (varios picos exotérmicos acompañados de pérdidas de peso entre $350-600^{\circ} \mathrm{C}$ ) (21) y grafito (pico exotérmico acompañado de pérdidas de peso con máximo $\approx 900^{\circ} \mathrm{C}$ ) en todos los materiales. Así mismo, se puede ver claramente en la muestras AMC1 y AMC3 un pico endotérmico muy fino $\mathrm{a} \approx 660^{\circ} \mathrm{C}$, correspondiente a la fusión del Al metálico. En los tres materiales se registra la presencia de distintos picos exotérmicos acompañados de ganancias de peso a $\mathrm{T}>1000^{\circ} \mathrm{C}$.

A partir de los datos obtenidos por ATD-TG se han definido las temperaturas de los tratamientos térmicos conducentes a oxidar ciertos componentes del refractario para su posterior análisis por las técnicas de FRX e ICP-OES.

A la temperatura de $600{ }^{\circ} \mathrm{C}$, se elimina la resina utilizada como ligante y el grafito, y se evita la fusión del aluminio metálico que ocurre a $660{ }^{\circ} \mathrm{C}$. A $1100{ }^{\circ} \mathrm{C}$ se oxida totalmente el $\mathrm{Al}$ metálico a alúmina $\mathrm{y} / \mathrm{o} \mathrm{MgAl}_{2} \mathrm{O}_{4}$.

En la Figura 4 se muestran los difractogramas de los materiales tratados térmicamente a $600{ }^{\circ} \mathrm{C} / 34 \mathrm{~h}$ y $1100{ }^{\circ} \mathrm{C} / 22 \mathrm{~h}$.
TABLA II. COMPONENTES UTILIZADOS EN LA FORMULACIÓN DE LOS REFRACTARIOS (MOLR Y DRX).

\begin{tabular}{|l|c|c|c|}
\hline Tipo de agregado & AMC1 & AMC2 & AMC3 \\
\hline Alúmina tabular & mayoritaria & mayoritaria & mayoritaria \\
Alúmina electrofundida & intermedia & intermedia & intermedia \\
marrón & - & - & minoritaria \\
Bauxita & minoritaria & intermedia & minoritaria \\
Periclasa $(\mathrm{MgO})$ & minoritaria & minoritaria & minoritaria \\
Grafito $(\mathrm{C})$ & minoritaria & minoritaria & minoritaria \\
Aluminio $(\mathrm{Al})$ & \multicolumn{3}{|r}{}
\end{tabular}
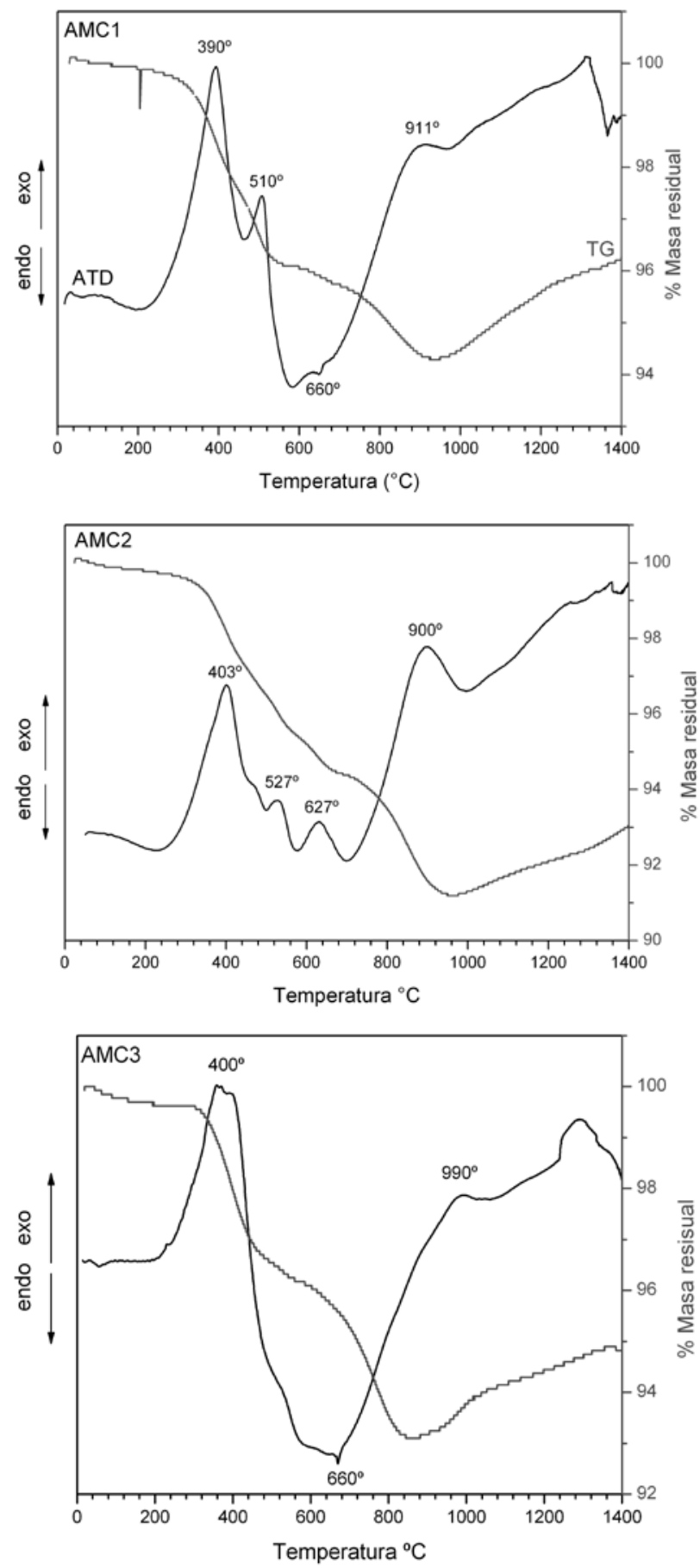

Figura 3. Curvas de análisis térmico diferencial y termogravimétrico de los refractarios. 
En dichos difractogramas se verifica la desaparición del pico más intenso del grafito $\left(26,38{ }^{\circ} 2 \theta\right.$; Ficha $\left.41-1487\right)$ a ambas temperaturas y del aluminio $\left(38,47^{\circ} 2 \theta\right.$; Ficha 04-0787) en el polvo tratado a $1100{ }^{\circ} \mathrm{C}$. Adicionalmente, se observa la formación de espinela en las muestras tratadas a 1100 ${ }^{\circ} \mathrm{C}$ (36,71 ${ }^{\circ} 2 \theta$; Ficha 75-1798). Teniendo en cuenta estos resultados, los picos exotérmicos a $\mathrm{T}>1000^{\circ} \mathrm{C}$ que se observan en el termograma de ATD (Figura 3) pueden atribuirse a la oxidación del Al seguida de reacción de las fases oxidadas $\left(\mathrm{AlO}(\mathrm{g}), \mathrm{Al}_{2} \mathrm{O}_{3}(\mathrm{~s})\right)$ con el $\mathrm{MgO}$ para formar espinela $\left(\mathrm{MgAl}_{2} \mathrm{O}_{4}\right)$, según reacciones sólido-gas y sólido-sólido [22].

A partir de los termogramas se han definido las temperaturas de los tratamientos térmicos con el fin de oxidar selectivamente la resina y el grafito para su cuantificación: 500 ${ }^{\circ} \mathrm{C}$ para eliminar la resina (por carbonización y oxidación del carbono residual) y $800^{\circ} \mathrm{C}$ para, además, oxidar completamente al grafito. Los termogramas obtenidos por TG del material
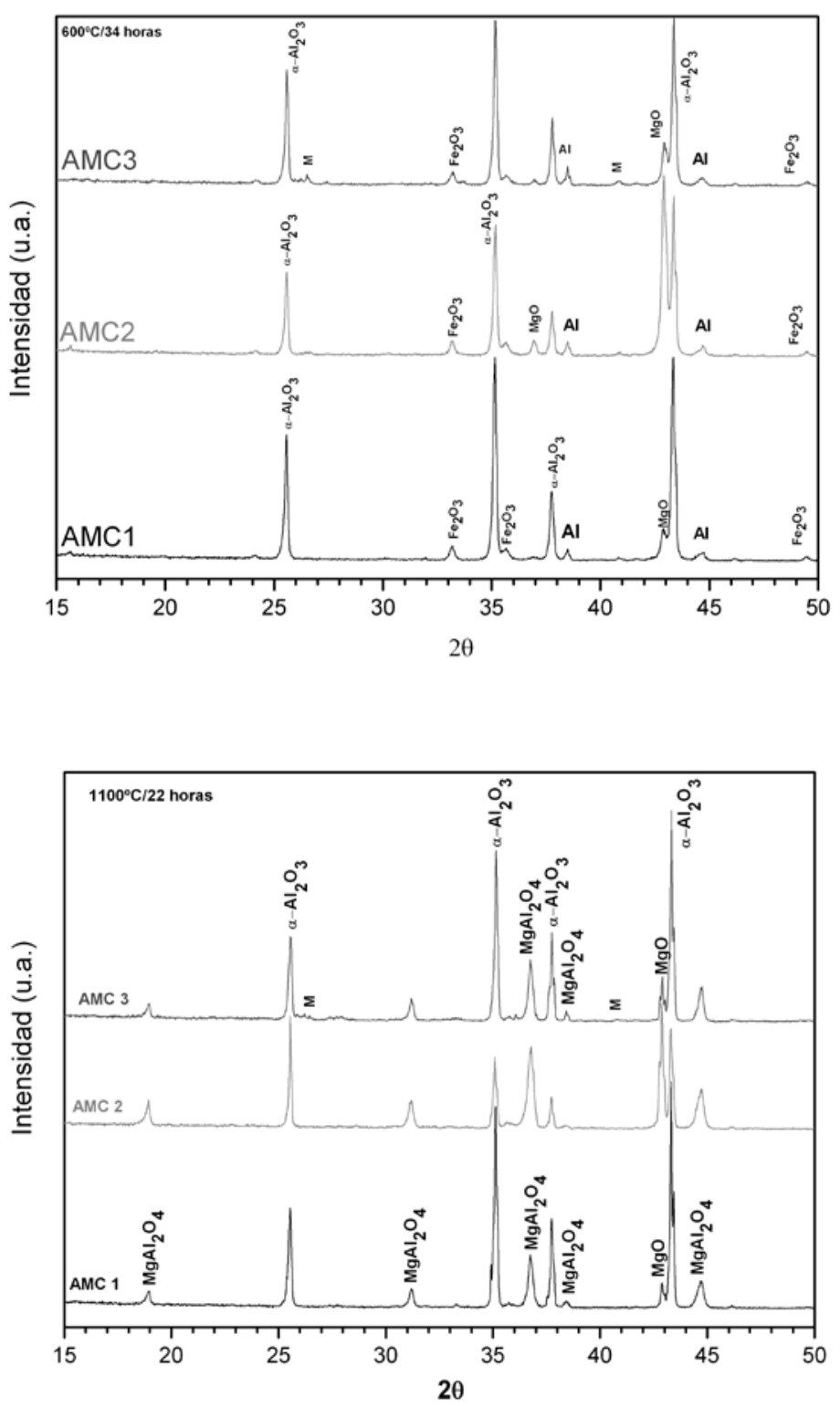

Figura 4. Difractogramas de los materiales tratados a $600{ }^{\circ} \mathrm{C} / 34 \mathrm{~h}$ y $1100^{\circ} \mathrm{C} / 22 \mathrm{~h}$. original y de los polvos tratados a 500 y $800{ }^{\circ} \mathrm{C}$ durante 4 $\mathrm{h}$ se presentan en la Figura 5 para el refractario AMC1. Los restantes materiales presentaron una respuesta similar frente a los respectivos tratamientos térmicos. En el polvo tratado a $500{ }^{\circ} \mathrm{C}$ no se registra la pérdida de peso a temperaturas < $700{ }^{\circ} \mathrm{C}$, atribuida a la transformación de la resina, aunque persiste la que ocurre entre $\approx 670$ y $850{ }^{\circ} \mathrm{C}$ correspondiente a la oxidación del grafito. Después del tratamiento a $800{ }^{\circ} \mathrm{C}$ no se registró ninguna pérdida de peso. Estos hechos confirman la efectividad de los tratamientos térmicos para eliminar en forma separada la resina y el grafito.

\subsection{Análisis químico}

Para cuantificar los componentes inorgánicos de los materiales refractarios estudiados se ha empleado la técnica de FRX. Las muestras a estudiar se han tratado a $1100{ }^{\circ} \mathrm{C} / 22 \mathrm{~h}$ para eliminar la resina y el grafito, y en este proceso el $\mathrm{Al}^{0}$ se ha transformado completamente en $\mathrm{Al}_{2} \mathrm{O}_{3}$ y posteriormente en espinela según la reacción: $4 \mathrm{Al}^{0}(\mathrm{~s})+2 \mathrm{MgO}(\mathrm{s})+3 \mathrm{O}_{2}(\mathrm{~g}) \rightarrow$ $2 \mathrm{MgAl}_{2} \mathrm{O}_{4}(\mathrm{~s})$.

$\mathrm{El}$ análisis químico de estas muestras se ha realizado empleando curvas de calibrado obtenidas a partir 21 patrones certificados de materiales silico-aluminosos, en un rango de concentraciones para los elementos mayoritarios, de $\mathrm{Al}_{2} \mathrm{O}_{3}$ : 0,16-88,8 \% en peso; $\mathrm{MgO}: 0,96-43,5 \%$ en peso.

La técnica de FRX mide el aluminio total de la muestra, independientemente del estado de oxidación en el que se encuentre.

La cantidad de $\mathrm{Al}_{2} \mathrm{O}_{3}$ total determinada en esta muestra incluye fundamentalmente, al corindón $\left(\alpha-\mathrm{Al}_{2} \mathrm{O}_{3}\right)$ de las diferentes fracciones granulométricas más el correspondiente a la oxidación del aluminio. La diferencia entre la cantidad total de $\mathrm{Al}_{2} \mathrm{O}_{3}$ y la que proviene del aluminio metálico (obtenida por estequiometría a partir del contenido de $\mathrm{Al}^{0}$ determinado por ICP-OES), equivale a la cantidad de alúmina de la muestra como corindón.

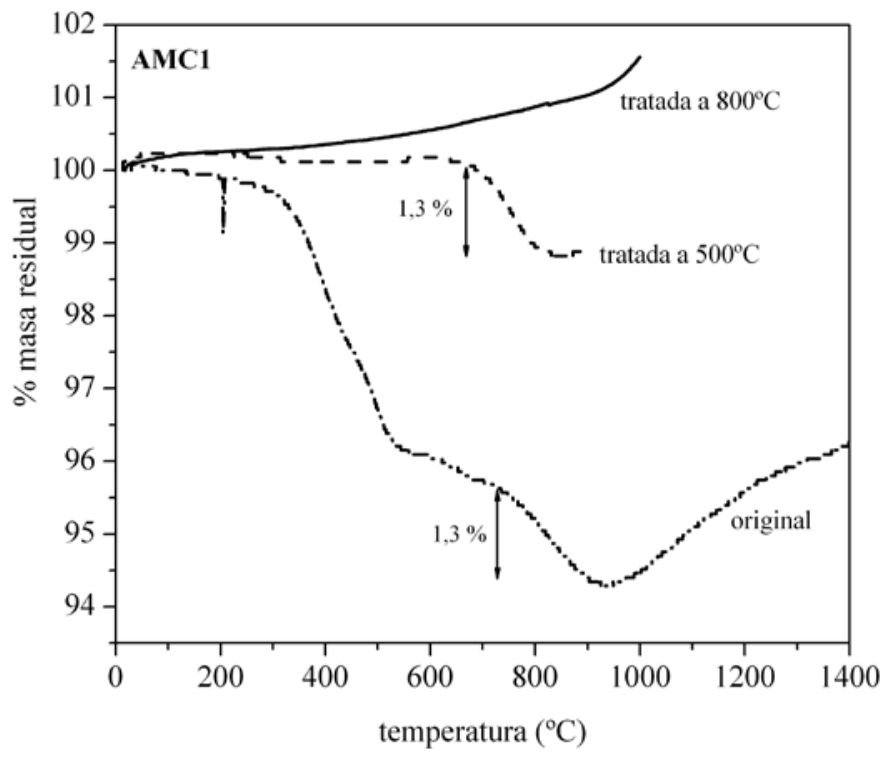

Figura 5. Termograma (TG) del polvo del material original y tratado a 500 y $800^{\circ} \mathrm{C}(\mathrm{AMC} 1)$. 
En la Figura 6 se muestra un esquema de la metodología utilizada para los análisis de los materiales AMC estudiados. Los resultados del análisis por FRX e ICP-OES expresados en porcentajes en peso de óxidos (con su desviación estándar calculada a partir de cinco repeticiones), junto a las proporciones de resina y grafito obtenidos por gravimetría, se recogen en la tabla III.

El contenido de aluminio resulta muy similar en los tres materiales, así como también el porcentaje en peso de resina. Sin embargo, el porcentaje de grafito es algo menor en AMC1 respecto a los otros dos refractarios. La cantidad de magnesia en AMC2 es alrededor de 5 veces mayor que en los restantes materiales; a este hecho se atribuye el incremento del contenido de $\mathrm{Fe}_{2} \mathrm{O}_{3^{\prime}} \mathrm{SiO}_{2^{\prime}} \mathrm{CaO}$ y otras impurezas minoritarias en AMC2, con respecto a AMC1. El mayor porcentaje de grafito también puede contribuir al aumento de $\mathrm{SiO}_{2^{\prime}}$ el cual forma parte de las cenizas provenientes de las arcillas que suele tener el grafito como impurezas.

En AMC3, el contenido de alúmina libre resultó inferior a AMC1 por la presencia de mullita en los agregados de bauxita (confirmados por microscopía óptica), que se corresponde con la mayor proporción de $\mathrm{SiO}_{2}$ en este material. Los contenidos de $\mathrm{Fe}_{2} \mathrm{O}_{3}$ y $\mathrm{TiO}_{2}$ se incrementaron en el orden: AMC1 < AMC2 < AMC3; entre AMC1 y AMC2, esto podría interpretarse como una mayor proporción de alúmina electrofundida marrón, mientras que el incremento en AMC3 se atribuye fundamentalmente a la presencia de bauxita.

En relación a otras fases minoritarias, el $\mathrm{P}_{2} \mathrm{O}_{5}$ se encuentra posiblemente como aditivo de la resina y el $\mathrm{CaO}$ acompaña principalmente a la periclasa, como silicato cálcico y/o vidrio. El contenido total de impurezas minoritarias sigue el orden: AMC1 $(1,64 \%)<\operatorname{AMC} 2(2,84 \%)<$ AMC3 $(4,90 \%)$, que resulta consistente con mayores porcentajes de $\mathrm{MgO}$ sinterizada y alúmina electrofundida (corindón marrón) en AMC2 y de bauxita en AMC3.

Los resultados obtenidos por FRX, ICP y gravimetría (Tabla III) se interpretaron conjuntamente con los obtenidos del análisis microestructural y de DRX con el fin de establecer el análisis racional de los tres ladrillos $\mathrm{Al}_{2} \mathrm{O}_{3}-\mathrm{MgO}-\mathrm{C}$ estudiados, los cuales se enumeran a continuación:

- en los tres materiales, la alúmina se encuentra como corindón $\left(\alpha-\mathrm{Al}_{2} \mathrm{O}_{3}\right)$, en todos los agregados (tabular, electrofundida, bauxita) y el $\mathrm{MgO}$ como periclasa.

- el $\mathrm{Fe}_{2} \mathrm{O}_{3}$ se encuentra principalmente como: a) hematita, acompañando a la magnesia y la bauxita (en AMC3), b) titanatos $\left(\mathrm{Al}_{2-\mathrm{x}} \mathrm{Fe}_{\mathrm{x}} \mathrm{TiO}_{5}\right)$ principalmente en la bauxita (en AMC3) y c) formando soluciones sólidas en $\mathrm{MgO}$ y $\mathrm{Al}_{2} \mathrm{O}_{3^{\prime}}$; en cualquiera de los casos, no es posible discriminar cuánto del contenido total de hierro (determinado como $\mathrm{Fe}_{2} \mathrm{O}_{3}$ ) está presente de una $\mathrm{u}$ otra forma.

- en AMC1 y AMC2, las impurezas minoritarias de $\mathrm{SiO}_{2}$ se encuentran acompañando a los agregados de magnesia, en forma de silicatos cálcicos y/o de magnesio, y/o vidrio, y al grafito, donde está como cenizas provenientes de las impurezas de arcilla

- en AMC3, el $\mathrm{SiO}_{2}$ se encuentra, además, formando parte de la bauxita como mullita $\left(3 \mathrm{Al}_{2} \mathrm{O}_{3} \cdot 2 \mathrm{SiO}_{2}\right)$, por lo cual se asume que todo el $\mathrm{SiO}_{2}$ forma parte de esta fase por medio de la reacción: $3 \mathrm{Al}_{2} \mathrm{O}_{3}+2 \mathrm{SiO}_{2} \rightarrow 3 \mathrm{Al}_{2} \mathrm{O}_{3} \cdot 2 \mathrm{SiO}_{2}$; a partir de esta suposición se calcula la cantidad de $\mathrm{Al}_{2} \mathrm{O}_{3}$ que forma mullita, y se descuenta del contenido total de este óxido como corindón.

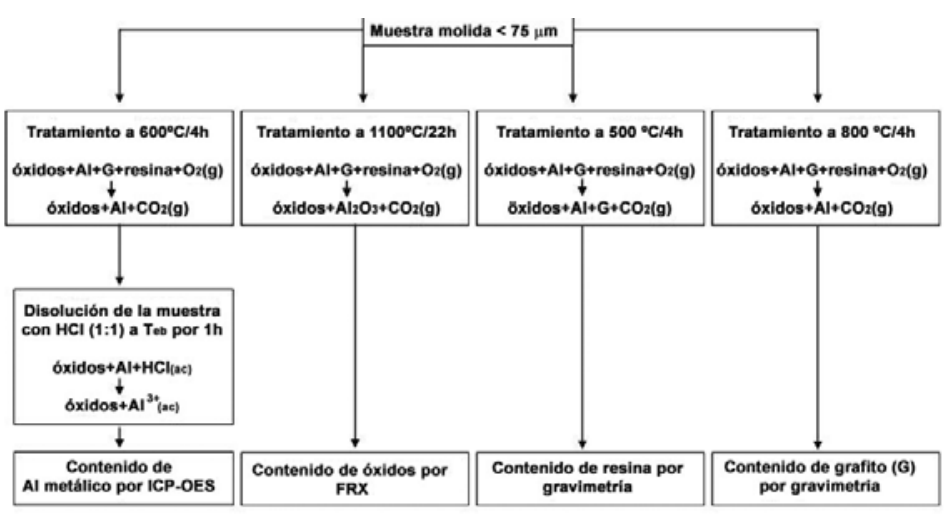

Figura 6. Esquema del método de análisis utilizado.

TABLA III. ANÁLISIS QUÍMICO (\% PESO) DE LOS REFRACTARIOS $\mathrm{Al}_{2} \mathrm{O}_{3}$-MgO-C. EL ERROR CORRESPONDE A LA DESVIACIÓN ESTÁNDAR CALCULADA A PARTIR DE CINCO REPETICIONES

\begin{tabular}{|c|c|c|c|c|}
\hline Componente & $\mathrm{AMC} 1$ & $\mathrm{AMC} 2$ & AMC3 & Técnica \\
\hline $\mathrm{Al}_{2} \mathrm{O}_{3}$ & $82,7 \pm 0,3$ & $57,6 \pm 0,3$ & $76,6 \pm 0,3$ & FRX \\
$\mathrm{Al}^{\circ}$ & $1,39 \pm 0,02$ & $1,37 \pm 0,02$ & $1,60 \pm 0,02$ & ICP-OES \\
$\mathrm{Fe}_{2} \mathrm{O}_{3}$ & $1,59 \pm 0,02$ & $1,99 \pm 0,02$ & $2,07 \pm 0,02$ & FRX \\
$\mathrm{MgO}$ & $5,40 \pm 0,03$ & $26,9 \pm 0,1$ & $6,80 \pm 0,03$ & FRX \\
$\mathrm{SiO}_{2}$ & $0,68 \pm 0,01$ & $1,16 \pm 0,01$ & $2,42 \pm 0,01$ & FRX \\
$\mathrm{K}_{2} \mathrm{O}$ & - & - & $0,072 \pm 0,005$ & FRX \\
$\mathrm{P}_{2} \mathrm{O}_{5}$ & $0,016 \pm 0,002$ & $0,040 \pm 0,004$ & $0,075 \pm 0,004$ & FRX \\
$\mathrm{CaO}$ & $0,14 \pm 0,01$ & $0,52 \pm 0,01$ & $0,30 \pm 0,01$ & FRX \\
$\mathrm{Cr}_{2} \mathrm{O}_{3}$ & $0,090 \pm 0,02$ & $0,10 \pm 0,02$ & $0,15 \pm 0,02$ & FRX \\
$\mathrm{TiO}_{2}$ & $0,50 \pm 0,01$ & $0,80 \pm 0,01$ & $1,50 \pm 0,02$ & FRX \\
$\mathrm{MnO}$ & $0,024 \pm 0,002$ & $0,038 \pm 0,002$ & $0,025 \pm 0,002$ & FRX \\
$\mathrm{ZrO}$ & $0,031 \pm 0,003$ & $0,070 \pm 0,003$ & $0,20 \pm 0,01$ & FRX \\
$\mathrm{SrO}$ & $0,029 \pm 0,003$ & $0,041 \pm 0,003$ & $0,081 \pm 0,003$ & FRX \\
$\mathrm{ZnO}$ & $0,049 \pm 0,004$ & $0,052 \pm 0,004$ & - & FRX \\
$\mathrm{Grafito}_{2}$ & $1,7 \pm 0,1$ & $3,5 \pm 0,1$ & $3,0 \pm 0,1$ & Gravimetría \\
$\mathrm{Resina}$ & $5,4 \pm 0,1$ & $5,6 \pm 0,1$ & $5,0 \pm 0,1$ & Gravimetría \\
\hline
\end{tabular}

TABLA IV: COMPOSICIÓN MINERALÓGICA (\% EN PESO) DE LOS REFRACTARIOS, OBTENIDA MEDIANTE UN ANÁLISIS RACIONAL.

\begin{tabular}{|l|c|c|c|}
\hline Fases & AMC1 & AMC2 & AMC3 \\
\hline $\mathrm{Al}_{2} \mathrm{O}_{3}$ & 82,7 & 57,6 & 70,5 \\
\hline $\mathrm{Al}^{0}$ & 1,4 & 1,4 & 1,6 \\
\hline $\mathrm{MgO}$ & 5,4 & 26,9 & 6,8 \\
\hline $\mathrm{Fe}_{2} \mathrm{O}_{3}$ & 1,6 & 2,0 & 2,0 \\
\hline $3 \mathrm{Al}_{2} \mathrm{O}_{3} \cdot 2 \mathrm{SiO}_{2}$ & - & - & 8,6 \\
\hline $\mathrm{C}$ (grafito) & 1,7 & 3,5 & 3,0 \\
\hline resina & 5,4 & 5,6 & 5,0 \\
\hline otras impurezas & 1,6 & 2,8 & 2,7 \\
\hline
\end{tabular}


Sobre la base de estas consideraciones, se determinaron los porcentajes de las principales fases presentes en los tres refractarios AMC, indicados en la Tabla IV. En relación al $\mathrm{TiO}_{2}$, cuyo porcentaje está incluido en "otras impurezas", su presencia está asociada principalmente, aunque no exclusivamente, a la bauxita; en el análisis por DRX de AMC3, el único material que contiene bauxita, se identifico más de una fase con titanio, $\mathrm{Al}_{2 \mathrm{x}} \mathrm{Fe}_{\mathrm{x}} \mathrm{TiO}_{5}$ (titanatos de $\mathrm{Al}$ ó Fe, con $\mathrm{Fe}$ o $\mathrm{Al}$ en solución sólida, respectivamente) y $\mathrm{TiO}_{2}$ (rutilo). $\mathrm{Al}$ igual que en el caso del hierro, no es posible determinar la contribución de cada una de estas fases, aunque teniendo en cuenta el contenido total de $\mathrm{TiO}_{2}(1,56 \%)$, se puede estimar que están presentes en proporciones $<1 \%$ en peso.

El análisis racional de los resultados obtenidos mediante análisis químico, térmico y difracción de rayos $\mathrm{X}$ cualitativa es una herramienta sencilla, práctica y precisa (con un error $\pm 5 \%$ en peso) para la determinación cuantitativa de la composición mineralógica de los materiales de $\mathrm{MgO}-\mathrm{Al}_{2} \mathrm{O}_{3}-\mathrm{C}$. Los resultados obtenidos mediante análisis racional pueden cuantificarse experimentalmente por DRX utilizando el método de Rietveld (19), aunque este es un procedimiento complejo que requiere usuarios con un entrenamiento específico.

\section{CONCLUSIONES}

En este trabajo se propone una metodología completa para caracterizar la composición química y mineralógica de refractarios de $\mathrm{Al}_{2} \mathrm{O}_{3}-\mathrm{MgO}-\mathrm{C}$.

La elevada consistencia entre los resultados obtenidos por el uso combinado y estratégico de la microscopia óptica de luz reflejada, difracción de rayos $X$, análisis térmico diferencial y termogravimétrico, fluorescencia de rayos $X$, espectroscopia de emisión de plasma y gravimetría atestigua la exactitud del método propuesto para la caracterización de refractarios multicomponentes. El análisis térmico diferencial permite discriminar la pérdida de peso debida al grafito de la procedente de la combustión de la resina.

Los resultados obtenidos de la combinación de los diferentes estudios y la precisión del método propuesto para cada elemento analizado, y la precisión de cada método de determinación utilizado son aceptables.-

El protocolo establecido es más simple y posee una precisión equivalente en los datos experimentales a la de otras metodologías establecidas previamente.

\section{FUENTES DE FINANCIACIÓN}

Este trabajo se sido financiado a través de los proyectos "Degradación termoquímica y termomecánica de refractarios óxido-C de uso siderúrgico" (PICT2006 N 1887), otorgado por la Agencia Nacional de Promoción Científica y Tecnológica (ANPCyT) de Argentina y proyecto MAT2010-17753 del Ministerio de Ciencia e Innovación de España. Así mismo, este trabajo se inscribe en los trabajos de la Red CYTED "Hormigones Refractarios" (P311RT0035).

\section{REFERENCIAS}

1. S. Miglani, J. J Uchno, "Resin bonded alumina-magnesia-carbon brick for ladles". Proceeding of UNITECR'97 (New Orleans, USA), 193-201 (1997).

2. R.K. Koley, A.V. Rao, S. Askar, S.K. Srivastava, "Development and application of $\mathrm{Al}_{2} \mathrm{O}_{3}-\mathrm{MgO}-\mathrm{C}$ refractory for secondary refining ladle", Proceeding of UNITECR'01 (Cancún, México), 2001.

3. A. D. Gupta, K. Vickram, "Development of resin-bonded aluminamagnesia-carbon bricks for steel ladle applications", Interceram, Vol. 48, 5, 307-310 (1999).

4. M. Kamiide, S. Yamamoto, K. Yamamoto, K. Nakahara, N. Kido, “Damage of $\mathrm{Al}_{2} \mathrm{O}_{3}-\mathrm{MgO}-\mathrm{C}$ brick for ladle furnace", J. Tech. Assoc. Refract., Japan, 21, 252-257 (2001).

5. A.A. Nourbakhsh, Sh. Salarian, S.M. Hejazi, S. Shojaiei, F. Golestani-Fard, "Increasing durability of ladle lining refractories by utilizing $\mathrm{Al}_{2} \mathrm{O}_{3}-\mathrm{MgO}-\mathrm{C}$ bricks", Proceeding of UNITECR'03 (Osaka, Japan), 499-502 (2003).

6. L. Musante, V. Muñoz, M.H. Labadie, A.G. Tomba Martinez, "High temperature mechanical behavior of $\mathrm{Al}_{2} \mathrm{O}_{3}-\mathrm{MgO}-\mathrm{C}$ refractories for steelmaking use", Ceram. Int., 37, 1473-1483 (2011).

7. V. Muñoz, G.A. Rohr, A.G. Tomba Martínez, A.L. Cavalieri. “Aspectos experimentales de la determinación de curvas esfuerzo-deformación a alta temperatura y en atmósfera controlada: Refractarios $\mathrm{Al}_{2} \mathrm{O}_{3}-\mathrm{MgO}-\mathrm{C}^{\prime \prime}, \mathrm{Bol}$. Soc. Esp. Ceram. Vidr. Vol. 50, 3, 117-124. (2011).

8. P. Pena, A. H. De Aza, L. Contreras, R. García-Carrodeguas, S. De Aza, J. F. Almagro, C. Luna, M. Guerrero Barranco, F. Javier Ferrer, "Mecanismo de corrosión a refractarios de $\mathrm{MgO}-\mathrm{C}$ y MgO-C-Al en horno eléctrico". Bol. Soc. Esp. Ceram. Vidr., 49 (3) 157-176 (2010).

9. C.F. Cooper, I.C. Alexander, C.J. Hampson, "The role of graphite in the thermal shock resistance of refractories", Br. Ceram. Trans. J., 84, 57-62 (1985).

10. Cooper, C. F., "Refractory Application of Carbon," Brit. Ceram. Trans. J., 84, 48-53 (1985).

11. C. Baudín, C. Alvarez, R.E. Moore, "Influence of Chemical Reactions in Magnesia-Graphite Refractories: Part I, Effects on Texture and HighTemperature Mechanical Properties", J. Am. Ceram. Soc., 82 [12] 3529-38 (1999).

12. C. Baudín, C. Alvarez, R.E. Moore, "Influence of Chemical Reactions in Magnesia-Graphite Refractories: II, Effects of Aluminum and Graphite Contents in Generic Products", J. Am. Ceram. Soc., 82 [12] 3539-48 (1999).

13. G. Buchebner, V. Samm, J. Rothch, "Latest developments in Magnesiacarbon Tricks", RHI Bulletin, 1, 23-28 (2011).

14. A. Yamaguchi, "Application of thermochemistry to refractories", en: J. Bennett y J.D. Smith (Eds.), Fundamentals of Refractory Technology, Ceramic Transactions (Vol 125), The American Ceramic Society, 2001, pp. 157-170.

15. C. Taffin, J. Poirier, "The behaviour of metal additives in MgO-C and $\mathrm{Al}_{2} \mathrm{O}_{3}-\mathrm{C}$ refractories", Interceram, 354-358 [5]/458-460 [6] 43 (1984).

16. C. Baudín, "High temperature mechanical behaviour of magnesia graphite refractories", en: J. Bennett y J.D. Smith (Eds),.Fundamentals of Refractory Technology, Ceramic Transactions (Vol 125), The American Ceramic Society, Westerville, 2001, pp. 73-92.

17. M. Bavand-Vandchali, F. Golestani-Fard, H. Sarpoolaky, H.R. Rezai, C.G. Aneziris, "The influence of in situ spinel formation on microstructure and phase evolution of MgO-C refractories", J. Eur. Ceram. Soc., 28, 563-569 (2008).

18. H. Asakura. "X-Ray fluoresence spectrometry for refractories", J. Tech. Assoc. Refrac., Japan, 23 [2] 86-94 (2003).

19. A. H. De Aza, F. J. Valle, P. Ortega, P. Pena, S. De Aza, “Analytical Characterization of a Magnesia-Graphite Refractory," J. Am. Ceram. Soc., 89 [5] 1704-1708 (2006).

20. Á. G. De la Torre, F. J. Valle, A. H. De Aza, “Direct mineralogical composition of a MgO-C refractory material obtained by Rietveld methodology", J. Eur. Ceram. Soc., 26 [13] 2587-2592 (2006).

21. G. Jecko and D. Ravaine, "L' analyse des réfractaires modernes", Rev. Metall., 84 [1] 33-46 (1987).

22. S. Serena, MA. Sainz, A. Caballero "Experimental and thermodynamic analysis of the $\mathrm{Al} / \mathrm{C}$ ratio on the microchemistry and microstructure of MgO-C-Al materials" Bol. Soc. Esp. Ceram. V.46[6] 289-298 (2007).

23. K. Kanno, N. Koike, Y. Korai, I. Mochida, M. Komatsu, "Mesophase pitch and phenolic resin blends as binders for magnesia-graphited bricks", Carbon, 37, 195-201 (1999).

Recibido: $14 / 12 / 2011$

Aceptado: 30/11/2012 